\title{
Progress toward the chiral regime in lattice QCD calculations of the neutron electric polarizability
}

\section{Michael Engelhardt ${ }^{* i}$}

Department of Physics, New Mexico State University, Las Cruces

E-mail: engel@ nmsu. edu

The static electric polarizability of the neutron is evaluated using domain-wall valence quarks on a MILC asqtad sea quark ensemble corresponding to a pion mass of $m_{\pi}=357 \mathrm{MeV}$. Both connected as well as disconnected contributions are included. The result is quantitatively compatible with the expectation from chiral effective theory.

The XXVII International Symposium on Lattice Field Theory

July 26-31, 2009

Peking University, Beijing, China

*Speaker.

${ }^{\dagger}$ Supported by the U.S. DOE under grant DE-FG02-96ER40965. 


\section{Introduction}

Electromagnetic polarizabilities are fundamental properties of hadrons which figure prominently, e.g., in soft Compton scattering. In the low energy limit, when the wavelength of the scattered photons is large compared to the hadron size, the Compton scattering amplitude can be cast in terms of a gradient expansion in the electromagnetic field [1]. The leading terms in this expansion are determined by the static electric and magnetic polarizabilities, which are defined via the hadron mass shift in constant external electric and magnetic fields, respectively. In this work, specifically the static electric polarizability of the neutron is investigated, building on earlier exploratory work [2]. By pushing the calculation down to a pion mass of $357 \mathrm{MeV}$, contact is established with chiral effective theory; the approach is fully dynamical, including all disconnected quark loop contributions. Related efforts have recently been reported by several groups, cf. [3-5].

\section{Expansion of the neutron two-point function}

In parallel to the Compton scattering amplitude alluded to above, the response of the neutron mass to an external electric field can be expanded both with respect to the magnitude of the electric field as well as its space-time variation. Here, only the leading term in this combined expansion is of interest,

$$
m(E)-m(0)=-\frac{\alpha}{2} E^{2}+\ldots,
$$

discarding all space-time derivatives of the electric field and terms higher than quadratic in the same $^{1}$. The electric polarizability $\alpha$ can then be extracted by evaluating the neutron two-point function, which determines the neutron mass, in the presence of a constant electric field; the twopoint function can furthermore be expanded in that field from the outset, keeping only quadratic terms. Consider decomposing the action into the zero external field term and the perturbation due to the external field, $S=S_{0}+S_{\text {ext }}$. Then the two-point function expands as

$$
\begin{array}{r}
\left\langle N_{\beta}(y) \bar{N}_{\alpha}(x)\right\rangle=\int[D U][D \psi][D \bar{\psi}] \exp \left(-S_{0}\right)\left(1-S_{\text {ext }}+S_{\text {ext }}^{2} / 2+\ldots\right) N_{\beta}(y) \bar{N}_{\alpha}(x) / \\
\int[D U][D \psi][D \bar{\psi}] \exp \left(-S_{0}\right)\left(1-S_{\text {ext }}+S_{\text {ext }}^{2} / 2+\ldots\right)
\end{array}
$$

As usual, the terms in the denominator cancel statistically disconnected parts of diagrammatically disconnected contributions. The coupling to the external electric field still remains to be specified. In the following, the electric field will be taken to point into the spatial 3-direction, and will be introduced via the 3-component of the gauge field, $A_{3}=E\left(t-t_{0}\right)$. This choice will be discussed in depth further below; it decisively influences the physics of the external field method calculation pursued here. For now, it is sufficient to remark that only $A_{3}$ is non-zero, that it is constant in the 3-direction, and that it is linear in $E$. Under these circumstances, the gauge links are modified by the external field as

$$
U_{3} \longrightarrow \exp \left(i q \int d x_{3} \cdot A_{3}\right) \cdot U_{3}=\left(1+i a q A_{3}-a^{2} q^{2} A_{3}^{2} / 2+\ldots\right) \cdot U_{3}
$$

\footnotetext{
${ }^{1}$ From the outset, the absence of a neutron electric dipole moment is assumed in the present treatment; this would of course no longer be accurate in the presence of a non-trivial vacuum $\Theta$-angle.
} 


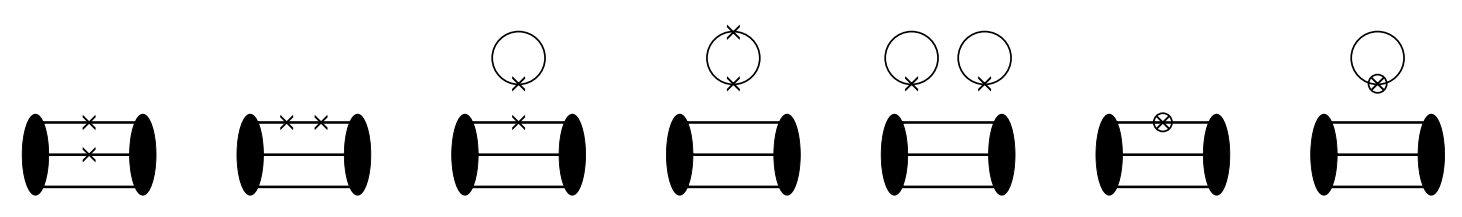

Figure 1: Diagrammatic representation of contributions to the neutron electric polarizability. Crosses denote interaction vertices linear in the external field, circled crosses interaction vertices quadratic in the same.

(where $a$ denotes the lattice spacing and $q$ the quark electric charge matrix) and, inserting this into fermion action discretizations of the Wilson type, the coupling to the external field takes the form ${ }^{2}$

$$
\begin{aligned}
S_{\text {ext }}=Z_{V} \cdot \frac{1}{2} \sum_{x} \bar{\psi}(x)\left[\left(i a q A_{3}-a^{2} q^{2} A_{3}^{2} / 2\right) \cdot U_{3}(x) \cdot\left(-1+\gamma_{3}\right) \cdot \psi\left(x+e_{3}\right)\right) & \\
& \left.\left.+\left(i a q A_{3}+a^{2} q^{2} A_{3}^{2} / 2\right) \cdot U_{3}^{\dagger}\left(x-e_{3}\right) \cdot\left(1+\gamma_{3}\right) \cdot \psi\left(x-e_{3}\right)\right)\right]
\end{aligned}
$$

Thus, two interaction vertices are generated, one linear in the external field, and one quadratic. Carrying out the fermionic integrations in (2.2), i.e., applying Wick's theorem, leads to these vertices being inserted into quark propagators, yielding the diagrammatic representation of the relevant contributions to the neutron two-point function depicted in Fig. 1.

\section{Role of the external gauge field}

As already mentioned in the previous section, the external electric field will be introduced via a non-vanishing 3-component of the gauge field,

$$
A_{3}=E\left(t-t_{0}\right) \equiv A+E t
$$

To arrive at a cogent interpretation of the neutron two-point function measured in the presence of such a field, a detailed consideration of the resultant physical effects is called for. First and foremost, note the freedom in the choice of the origin in the time direction, $t_{0}$, which is equivalent to a freedom of adding a constant, $A$, to the gauge field. If the spatial directions were infinite, this choice would be inconsequential, since $A$ could be freely shifted by a gauge transformation. However, on a finite space of linear extent $L$ with periodic boundary conditions, only a discrete gauge invariance $A \longrightarrow A+2 \pi / q_{0} L$ remains, where $q_{0}=e / 3$ represents the elementary electric charge present in the system. Apart from this residual invariance, physics, and, in particular, the mass shift of the neutron depends on the chosen value of $A$. The question therefore arises how the electric polarizability proper can be disentangled from the $A$-dependence of the neutron mass.

\footnotetext{
${ }^{2}$ The renormalization factor $Z_{V}$ is due to a technical detail: In the numerical calculation, quark lines in Fig. 1 will be populated by domain wall fields; however, the coupling to the external field is not realized via the five-dimensional conserved current, but via the current obtained by projecting the quark modes onto the four-dimensional domain walls. The fields $\bar{\psi}, \psi$ in (2.4) are the projected ones. This amounts to a renormalization of the vertices, which is compensated by $Z_{V}$. The renormalization factor is determined to be $Z_{V}=1.14$ for light quarks and $Z_{V}=1.10$ for strange quarks by measuring the number of valence quarks in baryons.
} 
Physically, $q A$ enters the quark Hamiltonian ${ }^{3}$ as a quark Bloch momentum in the periodic lattice. Thus, for general $A$, there is a current of up quarks flowing around the torus with each up quark, on the average, carrying momentum $2 e A / 3$, and a current of twice as many down quarks with, on the average, half as much momentum each in the opposite direction ${ }^{4}$. Only the complete (periodic) "neutron", if it can be viewed as such, maintains vanishing momentum. Of course, the notion of a neutron becomes tenuous when the Bloch momenta become large; in this limit, the system essentially consists of two strong, oppositely oriented quark electrical currents with some residual correlations due to the chromodynamic force. Certainly, any mass shift extracted in such a regime could hardly be construed as representing the polarizability of a reasonably isolated neutron. Instead, it is necessary to seek out the regime where the quark currents cease and a bona fide neutron, only weakly distorted by the electric field $E$ proper, is formed.

In the absence of an actual electric field, it is clear how to achieve this situation; one must set $A=0$. When $E \neq 0$, on the other hand, the situation is more complicated, since the Bloch momenta change (linearly) in time: In view of (3.1), a shift in the time direction is equivalent to a shift in $A$. This is, of course, entirely natural; the electric field accelerates the up and down quark electrical currents. A practical and model-independent way to identify when the quark currents cease is to look for stationarity in physical quantities such as the mass shift. If the quark currents are initially oriented opposite to the acceleration provided by the electric field, then time evolution will lead to a point at which the currents cease and turn around. Expanded around that point in time, the leading time dependence of physical quantities is quadratic. Equivalently, one may perform measurements of the neutron two-point function in a fixed time region, but for different values of $A$, and subsequently seek out the stationary point as a function of $A$. In practice, this calls for measurements at three different $A$, permitting the determination of a parabola in that variable, as well as its stationary point.

Before continuing the main line of discussion by turning to the extraction of the neutron mass shift from its two-point function, cf. section 4, a separate concern associated with the choice (3.1) of the external gauge field should be addressed. While this gauge field is constant, and thus periodic in the spatial directions, it must exhibit a discontinuity at some point in the time coordinate in order to be periodic in that direction as well. At the discontinuity, the electric field exhibits a spike, with the potential of distorting the measurement of the neutron mass shift, which is, after all, intended to be taken in a constant electric field. As demonstrated in [4], such an effect can indeed be seen if one insists on performing the mass measurement in a time region including the spike. For selected magnitudes of the electric field, the distortion can be avoided, because what is physically relevant is not the gauge field $A_{3}$ itself, but $e^{i q A_{3}}$; thus, if one arranges the discontinuity to be an appropriate multiple of $2 \pi$, it has no physical consequences. However, ultimately, this issue is moot, since nothing forces one to perform measurements near the gauge field discontinuity ${ }^{5}$. In

\footnotetext{
${ }^{3}$ In more precise terms, this would be an effective constituent quark Hamiltonian; the electrical current itself depends on the effective mass experienced by the charge carriers as they propagate with a given Bloch momentum.

${ }^{4}$ In addition to these valence currents, there are, of course, also analogous sea quark and antiquark currents.

${ }^{5}$ Note, however, that for the alternative choice of gauge field $A_{0}=E x_{3}$, the analogous objection would indeed be more serious; during the mass measurement, one cannot prevent the neutron from propagating to the spatial location of the electric field spike. Indeed, if one projects onto definite neutron momentum, all spatial positions are explicitly weighted equally.
} 
fact, in practice, it is not even necessary to extend the support of $A_{3}$ over all times; in the present investigation, just as in a real laboratory setting, the external field is merely switched on a little before the neutron source is introduced, and switched off some time after it is annihilated. This does not alter the result of the mass measurement, as tested in [2]; however, it significantly reduces the statistical fluctuations in disconnected diagrams, since quark loops far in the past and the future of (and thus not correlated with) the neutron are eliminated.

\section{Extracting the mass shift from the two-point function}

As should be clear from the discussion in the previous section, the neutron Hamiltonian in the presence of the external field adopted here is not time-independent; a shift in time corresponds directly to a shift in the constant $A$ in the external field (3.1). Before addressing the time dependence, it is useful to review how the mass shift would enter the neutron two-point function in the case of a time-independent Hamiltonian depending on two arbitrary small parameters $E$ and $A$. In such a case, projecting onto zero-momentum, unpolarized neutrons, the two-point function behaves as

$$
G(p=0, t)=\sum_{\vec{y}} \operatorname{Tr}\left(\frac{1+\gamma_{0}}{2}\langle N(y) \bar{N}(x)\rangle\right) \stackrel{t \rightarrow \infty}{\longrightarrow} W \exp (-m t)
$$

for large times, where both the overlap between neutron source and true ground state $W$ and the neutron mass $m$ can be expanded in $E$ and $A$,

$$
\begin{aligned}
W & =W_{0}+W^{(1)}(A, E)+W^{(2)}(A, E)+\ldots \\
m & =m_{0}+m^{(2)}(A, E)+\ldots
\end{aligned}
$$

Inserting this into (4.1), the term of second order in $E$ and $A$ in the two-point function becomes

$$
G^{(2)}(p=0, t) \stackrel{t \rightarrow \infty}{\longrightarrow} W_{0} \exp \left(-m_{0} t\right)\left(\frac{W^{(2)}(A, E)}{W_{0}}-m^{(2)}(A, E) t\right)
$$

implying that the neutron mass shift to second order in $E$ and $A$ can be obtained from (minus) the temporal slope of the correlator ratio $R(t)=G^{(2)}(t) / G^{(0)}(t)$.

Now, the Hamiltonian of interest here is

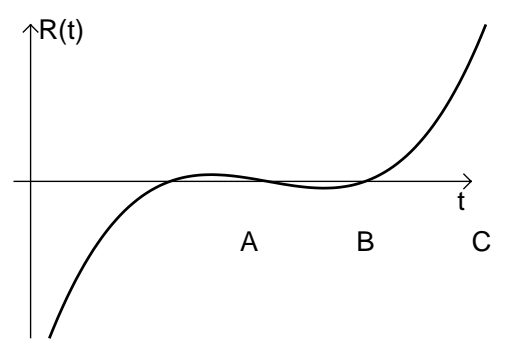

Figure 2: Generic behavior of the correlator ratio $R(t)=G^{(2)}(t) / G^{(0)}(t)$. Regions A, B and C qualitatively correspond to the time windows displayed in Fig. 3. not time-independent; however, if it is sufficiently weakly time-dependent, the system will adjust adiabatically while $W^{(2)}$ and $m^{(2)}$ vary slowly, and (4.4) still applies ${ }^{6}$, except that $W^{(2)}$ and $m^{(2)}$ are now time-dependent. This implies that $m^{(2)}$ cannot be simply read off as the slope of $R(t)$ anymore; this correlator ratio generically will behave as displayed in Fig. 2. However, as discussed further above, to obtain the electric polarizability of the neutron, one in particular needs

\footnotetext{
${ }^{6} \mathrm{~A}$ treatment beyond the adiabatic approximation, in the related context of the acceleration of a proton in an external electric field, has been described in [4].
} 

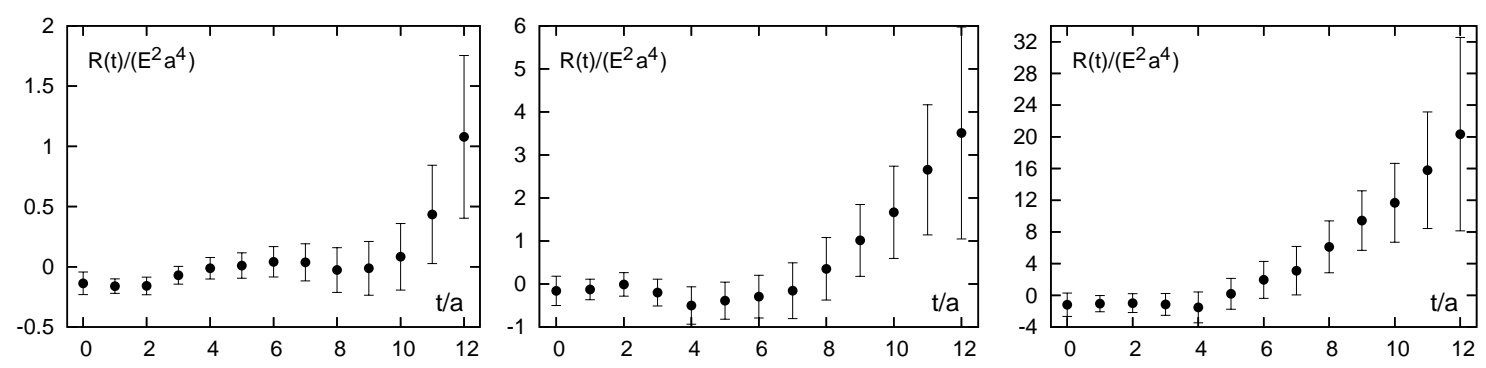

Figure 3: Correlator ratio $R(t)$ in (Gaussian) units of $E^{2} a^{4}$; in these units, the plots are independent of any actual value of $E$ used in the numerical computation. In the three measurements, the neutron source was placed at the fixed location $t=0$, and the background gauge field $A_{3}=E\left(t-t_{0}\right)$ was varied, with, from left to right, $t_{0}=6 a, t_{0}=0$ and $t_{0}=-10 a$. This generates time windows corresponding qualitatively, from left to right, to regions A, B and C in Fig. 2, respectively; note the variation in the vertical scale. The behavior indicated in Fig. 2 is thus demonstrated, with the inflection point, corresponding to the stationary point of the neutron Hamiltonian, being captured in the left panel.

only the mass shift at the stationary point in $A$, or, equivalently, $t$. Expanded around that point, the time dependence of $W^{(2)}$ and $m^{(2)}$ is quadratic; therefore, at that point, and only there, the mass shift $m^{(2)}$ is still given by (minus) the slope of $R(t)$, thus permitting the extraction of the neutron electric polarizability.

\section{Results}

Numerical measurements were carried out in a mixed action scheme, using 448 dynamical asqtad quark configurations provided by the MILC collaboration [6], with lattice spacing $a=0.124 \mathrm{fm}$ and quark masses $a m_{l}=0.01, a m_{s}=0.05$, corresponding to a pion mass of $357 \mathrm{MeV}$. Quark lines depicted in Fig. 1 were populated with domain wall quarks; all disconnected contributions were taken into account and were estimated using bulk complex $Z(2)$ stochastic sources. Fig. 3 displays three different measurements of the correlator ratio $R(t)=G^{(2)}(t) / G^{(0)}(t)$ performed with different shifts $A$, thus probing different time windows of the behavior displayed in Fig. 2. As a result of using Wuppertal smeared neutron sources and sinks optimized with respect to their overlap with the true neutron ground state, as constructed in [7], excited states are already negligible for $t \geq 4 a$ (where $t=0$ denotes the neutron source location). On the other hand, statistical uncertainties are under reasonable control up to $t=10 a$. In practice, the average slope of the correlator ratio $R(t)$ for $5 a \leq t \leq 9 a$ was determined in each of the three time windows displayed in Fig. 3; these three values define a parabola as a function of $A$, the extremum of which yields (minus) the mass shift at the stationary point, $-m^{(2)}\left(A_{\text {stat }}, E\right)$. The latter was corrected for curvature effects averaged over when determining the slope in each window. Alternative analysis schemes were explored and yield consistent results. Finally, supplementing $\left[-m^{(2)}\left(A_{\text {stat }}, E\right)\right] /\left(E^{2} a^{3}\right)$ with a factor 2 from (2.1) and a factor -1 from the continuation $E \rightarrow i E$ to Minkowski time yields the polarizability, in Gaussian units,

$$
\alpha=(0.9 \pm 1.6) \cdot 10^{-4} \mathrm{fm}^{3} .
$$

Fig. 4 puts this result in relation to chiral effective theory, specifically the small scale expansion (SSE) expectation [8], with which it is quantitatively compatible. Although the statistical uncer- 


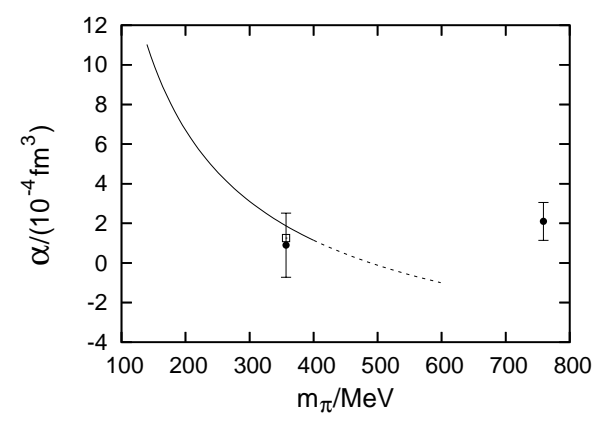

Figure 4: Electric polarizability of the neutron; solid circles with error bars denote lattice measurements from the present work (at $m_{\pi}$ $=357 \mathrm{MeV}$ ) and from [2] (at $m_{\pi}=759 \mathrm{MeV}$ ). The curve represents the SSE expectation [8], constructed purely from experimental data. Apart from the pion mass, which is varied in the graph, the SSE expression contains a collection of further parameters, among them the nucleon and delta masses, $f_{\pi}$ and $g_{A}$, cf. [8]. For these parameters, lattice measurements on the same ensemble at $m_{\pi}=357 \mathrm{MeV}$ exist; substituting those values into the SSE expression yields the open square. This can be viewed as the result of an alternative, partially resummed effective theory. tainty in (5.1) is sizeable due mainly to the disconnected contributions, the present calculation thus establishes contact with the pion mass regime in which chiral effective theory becomes applicable; an earlier exploratory study [2] at $m_{\pi}=759 \mathrm{MeV}$, the result of which is also included in Fig. 4, did not yet allow for a quantitative comparison ${ }^{7}$.

It is again worth emphasizing that, to arrive at the result (5.1), it was crucial to carefully take into account the physics associated with an external gauge field of the form (3.1). As explained in detail in section 3 , on a finite spatial lattice, the external field induces opposite Bloch momenta for the $u$ - and $d$-quarks, with a bona fide neutron only having an opportunity to form at a specific time, namely the stationary point at which the Bloch currents reverse direction under the influence of the electric field. That is where the electric polarizability can be extracted. The measurements displayed in Fig. 3 illustrate how, in a background field calculation of this type, the polarizability emerges as a rather small residual effect compared to the substantial Bloch momentum dependence.

\section{Acknowledgments}

NMCAC is acknowledged for providing the computing resources for this work on Encanto.

\section{References}

[1] D. Babusci, G. Giordano, A. I. L’vov, G. Matone and A. M. Nathan, Phys. Rev. C 58 (1998) 1013.

[2] M. Engelhardt, Phys. Rev. D 76 (2007) 114502.

[3] A. Alexandru and F. X. Lee, PoS LATTICE 2008 (2009) 145.

[4] W. Detmold, B. Tiburzi and A. Walker-Loud, PoS LATTICE 2008 (2009) 147.

[5] V. Guerrero, W. Wilcox and J. Christensen, PoS LATTICE 2008 (2009) 150.

[6] C. W. Bernard, T. Burch, K. Orginos, D. Toussaint, T. A. DeGrand, C. DeTar, S. Datta, S. A. Gottlieb, U. M. Heller and R. Sugar, Phys. Rev. D 64 (2001) 054506.

[7] D. Dolgov, R. Brower, S. Capitani, P. Dreher, R. G. Edwards, N. Eicker, U. Heller, T. Lippert, J. W. Negele, A. Pochinsky, D. Renner and K. Schilling, Phys. Rev. D 66 (2002) 034506.

[8] R. P. Hildebrandt, H. W. Grießhammer, T. R. Hemmert and B. Pasquini, Eur. Phys. J. A 20 (2004) 293.

\footnotetext{
${ }^{7}$ As already explained in a note added in [2], the result for the polarizability initially quoted there was missing an overall minus sign stemming from the continuation $E \rightarrow i E$ to Minkowski space.
} 